Arthroskopie $2017 \cdot 30: 274-275$

https://doi.org/10.1007/s00142-017-0165-7

Online publiziert: 13. September 2017

๑) Springer Medizin Verlag GmbH 2017

CrossMark

\section{P. Lobenhoffer ${ }^{1} \cdot$ F. Dirisamer ${ }^{2}$}

'Gelenkchirurgie Orthopädie, Hannover, Deutschland

${ }^{2}$ Orthopädische Praxis S. Shnayien, Linz am Rheim, Deutschland

\title{
Teilgelenkersatz am Knie
}

Liebe Leserinnen und Leser,

es ist Zeit, die operative Arthrosetherapie am Kniegelenk neu zu definieren. Aktivität ist ein Grundbedürfnis und wir sind als Operateure gehalten, Lebensfreude und Lebensqualität der Patienten zu sichern. Der bisherige Reflex - arthroskopische Interventionen, oftmals mehrfach, und dann beim Versagen eine Totalprothese, ist nicht mehr zeitgemäß.

\section{》) Es ist Zeit, die operative Arthrosetherapie am Kniegelenk neu zu definieren}

Es entspricht auch der aktualisierten Philosophie der AGA - Gesellschaft für Arthroskopie und Gelenkchirurgie, sich zunehmend auch um Methoden abseits der Arthroskopie zu bemühen. Rekonstruktive Eingriffe haben ihre natürlichen Grenzen. Totalendoprothesen stellen die Letztoption zur Versorgung der Gonarthrose dar. Dazwischen gibt es aber einen letztlich gut definierten Indikationsbereich, in dem der Gelenkerhalt mit einem Teilersatz kombiniert werden kann und soll - nach dem Motto: ersetzen was ersetzt werden muss, aber erhalten, was erhalten werden kann.

Die internationalen Prothesenregister haben gezeigt, dass die Ergebnisse der Knieendoprothetik bei jüngeren aktiven Patienten schlecht sind, dass die Erwartungen der Patienten in mindestens $20 \%$ der Fälle nicht erfüllt werden und dass die Revisionsrate inakzeptabel hoch ist $[1,3,4]$. Wir sollten eine patientenadaptierte und nicht eine prozedurenorientierte Versorgung anstreben. Die Daten aus den Prothesenregistern haben gezeigt, dass fast $50 \%$ der Arthrosepatienten eine einseitige Arthrose bei erhaltenem Bandapparat entwickeln [2]. Damit sind prinzipiell die Voraussetzungen für eine gelenkerhaltende Lösung gegeben. Unsere Zeitschrift hat bereits die knienahe Osteotomie bei Gonarthrose thematisiert. Nun ist es an der Zeit, auch den partiellen Gelenkersatz im Sinne der Schlittenprothese und der Femoropatellarprothese zu thematisieren. Es ist den Heftherausgebern ein Bedürfnis, darauf hinzuwirken, dass diese Lösungen künftig stärker Berücksichtigung finden. Das Risikoprofil des unikondylären Gelenkersatzes ist um den Faktor 2 bis 3 günstiger als jenes der Totalendoprothese, und die Ergebnisse aus Sicht der Patienten sind einhellig besser $[4,5]$. Diese Versorgungsstrategie erhält die einzigartige Kinematik des menschlichen Kniegelenks, erlaubt ein knochensparendes Arbeiten und erhält damit die Möglichkeit künftiger Revisionschirurgie. Der partielle Gelenkersatz ist technisch anspruchsvoll und erfordert profunde Kenntnisse der Anatomie und Biomechanik des Kniegelenks. Wichtig erscheint uns der Hinweis, dass sich in den Registerdaten der Trainingsgrad des Operateurs und seine persönliche Fallzahl als wesentliche Erfolgsfaktoren gezeigt haben. Der individuelle Chirurg sollte eine relevante Fallzahl pro Jahr erreichen, um gute Ergebnisse zu erzielen. Dies wird nach aller Erfahrung nur dann der Fall sein, wenn die Indikation zum unikondylären und femoropatellaren Gelenkersatz nicht auf Einzelfälle begrenzt wird, sondern systematisch in das Behandlungskonzept integriert ist. In diesem Sinne hoffen wir, Interesse für dieses Thema geweckt zu haben und die Verbreitung der gelenkerhaltenden Therapie der Gonarthrose voranzubringen.

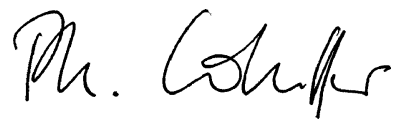

Philipp Lobenhoffer

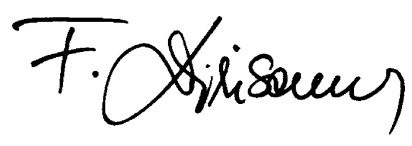

Florian Dirisamer

\section{Korrespondenzadresse}

Prof. Dr. med. P. Lobenhoffer

Gelenkchirurgie Orthopädie

Uhlemeyerstraße 16, 30175 Hannover,

Deutschland

philipp.lobenhoffer@g-o-hannover.de

Interessenkonflikt. P. Lobenhoffer und F. Dirisamer geben an, dass kein Interessenkonflikt besteht.

\section{Literatur}

1. Bayliss LE, Culliford D, Monk AP et al (2017) The effect of patient age at intervention on risk of implant revision after total replacement of the hip or knee: a population-based cohort study. Lancet 389(10077):1424-1430. https://doi.org/10.1016/ S0140-6736(17)30059-4

2. Hamilton TW, Pandit HG, Jenkins C et al (2017) Evidence-based indications for mobile-bearing unicompartmental knee arthroplasty in a consecutive cohort of thousand knees. J Arthroplasty 32(6):1779-1785. https://doi.org/10.1016/j.arth. 2016.12.036

3. Hunt LP, Ben-ShlomoY, Whitehouse MRet al (2017) The main cause of death following primary total hip and knee replacement for osteoarthritis: a cohort study of 26,766 deaths following 332,734 hip replacements and 29,802 deaths following 384,291 knee replacements. J Bone Joint Surg Am 99(7):565-575. https://doi.org/10.2106/JBJS.16. 00586

4. Liddle AD, Judge A, Pandit $\mathrm{H}$ et al (2014) Adverse outcomes after total and unicompartmental knee replacement in 101,330 matched patients: a study of data from the National Joint Registry for England and Wales. Lancet 384(9952):1437-1445. https:// doi.org/10.1016/S0140-6736(14)60419-0

5. Parvizi J, Nunley RM, Berend KR et al (2014) High level of residual symptoms in young patients after total knee arthroplasty. Clin Orthop Relat Res 472:133-137 
Hier steht eine Anzeige.

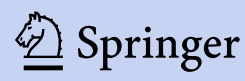

